Aus dem Neurologischen und dem Pharmakologischen Institut der Universität, Wien

\title{
Die Beeinflussung des Thetarhythmus im Kaninchenhippocampus durch elektrische Reizung von Septum und Formatio reticularis
}

\author{
Von \\ H.Petsche, G. GogoLāk, CH. StUMPF und T. NewkIR K * \\ Mit 4 Textabbildungen
}

(Eingegangen am 27. August 1963)

In früheren Untersuchungen (BRücke u. Mitarb. 1959a, b) wurde gezeigt, daß der beim Kaninchen im Hippocampus bei sensibler Reizung auftretende und mit Abflachung und Frequenzzunahme der corticalen Tätigkeit einhergehende Thetarhythmus (GreEn u. Ardurnr 1954) durch hochfrequente Reizung im Gebiet des Kernes des Brocaschen Bandes für die Dauer der Reizung unterdrückt werden kann. Diese Unterdrückung konnte auf eine reizbedingte Desynchronisierung der BurstAktivität der Septumzellen, welche eine conditio sine qua non für den Hippocampus-Thetarhythmus darstellt, zurückgeführt werden (PETsCHE, Gogolák u. STUMPF 1962). Wird dagegen mit derselben Reizqualität in der Formatio reticularis des Hirnstammes gereizt, kommt es zu einer Synchronisierung und Aktivierung des Thetarhythmus und erst bei supramaximaler Reizung zu seiner Aufhebung.

Langsame Reizung des Septums (ab etwa 4/sec) führt zum Auftreten einer Folge von Potentialen synchron der Reizung, was als ,ThetaDriving" bezeichnet wurde (BRÜCKE u. Mitarb. 1959a, b). Wie schon damals vermutet wurde, ist dieser Effekt auf aneinandergereihte evoked potentials zurückzuführen, die besonders deutlich dadurch hervortreten, daß der spontane Theta durch Reizung im Bereich seines pacemakers unterdrückt wird.

Bei langsamer Reizung der Formatio reticularis dagegen tritt ein eigenartiges Phänomen auf, dessen Klärung die folgende Untersuchung vorwiegend gewidmet ist: Wird mit einer Frequenz gereizt, die nur um ein geringes von der spontanen Thetafrequenz verschieden ist, so kommt es zu Modulationen, deren Frequenz der Differenz zwischen Reiz- und spontaner Thetafrequenz entspricht. An erster Stelle war zu klären, ob

*Derzeitige Addresse: University of Michigan, Ann Arbor, USA. 
dieses Phänomen ein rein physikalischer Überlagerungseffekt ist oder ob es zu einer darüber hinausgehenden Wechselwirkung zwischen retikulär ausgelösten rhythmischen evoked potentials und der Thetatätigkeit kommt, etwa analog den rhythmischen Schwankungen der corticalen Erregbarkeit, die CHAXG 1951 bei akustischen evoked potentials bei der Katze als vom Grundrhythmus abhängig nachweisen konnte. Daneben war die Frage zu entscheiden, durch welche Maßnahmen ein „resetting“ des Thetarhythmus erzeugt werden könnte, d.h. eine so tiefgreifende Änderung des Frequenzgefüges, daß es zu einer Neuordnung der Periodik durch den Reiz käme; und schließlich sollten die Experimente Aufschlüsse darüber geben, ob die durch Reizung der Formatio reticularis im Hippocampus ausgelösten Potentiale über denselben Weg geleitet werden wie die Impulse, die den Thetarhythmus veranlassen.

\section{Methodik}

Verwendet wurden 27 Kaninchen von durchschnittlich $2,5 \mathrm{~kg}$ Gewicht, die nach der von Monster modifizierten Hess-Technik präpariert wurden. Diese Technik wurde bereits ausführlich 1959 beschrieben (BRÜOKæ u. Mitarb. 1959b). Ein corticales Elektrodenpaar präzentral diente zur Kontrolle der corticalen Tätigkeit. Im Hippocampus befand sich eine achtfache Tiefenelektrode (acht Kontakte im Abstand von je 1,5 mm auf einer einzigen Trägernadel montiert). Die Lage der Pyramidenzellschicht im Bereich $\mathrm{CA}_{1}$ wurde aus der unipolaren Phasenumkehr ermittelt (GreEN u. Mitarb. 1960). Bipolare Reizelektroden befanden sich median im Septum und in der Formatio reticularis in Höhe des N. ruber. Als Reizquelle diente ein Grass- bzw. Tönnies-Stimulator. Gereizt wurde mit Rechteckimpulsen. (0,5 bzw. 0,05 msec, 250/sec und langsame Frequenzen im Thetabereich). Die Tiere waren nicht narkotisiert. Zur Eirzeugung eines kontinuierlichen Thetarhythmus wurde Eserin, $250 \gamma / \mathrm{kg}$, gegeben (SaILer u. STUMPE 1957). Registriert wurde uniund bipolar mit einem Sohwarzer-achtfach-EEG-Gerät.

\section{Ergebnisse}

Die Wirkung langsamer Reize im Septum auf den Hippocampusrhythmus wurden bereits ausführlich beschrieben (BRய̈CKE u. Mitarb. 1959a, b). Das dabei beobachtete „Theta-Driving“", d. h. das Auftreten von Potentialen, deren Frequenz von der Reizfrequenz abhing, konnte auf die Aktivierung von evoked potentials im Hippocampus bei unterdrücktem Thetarhythmus zurückgeführt werden.

Ein derartiges Phänomen läßt sich durch retikuläre Reizung nicht erzeugen, doch kommt es bei langsamer Reizung mit Frequenzen, die etwas von der Thetafrequenz abweichen, zu einer Amplitudenmodulation, die besonders deutlich und regelmäßig bei konstantem Theta ist. Dabei wechseln rhythmisch Zonen von regelrechter Thetatätigkeit mit Zonen, in denen die Thetawelle durch ein Spitzenpotential in zwei Komponenten gespalten ist (Abb.1). Bei rascher Registrierung $(6 \mathrm{~cm} / \mathrm{sec})$ kann man bei regelmäßigem Thetarhythmus unter Eserin beobachten, 
wie die Spitze, d.h. die deutlichste Komponente des evoked potential, langsam über die Thetawelle wandert. Modulationsfrequenzen bis zu etwa $0,1 / \mathrm{sec}$ (Perioden von 10 sec Dauer) konnten über Minuten beobachtet werden; d. h. die Thetafrequenz kann unter Eserin für einige Zeit

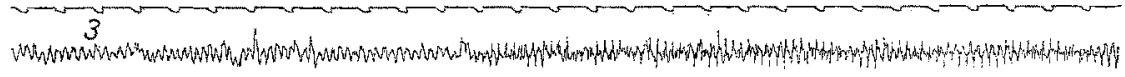
4

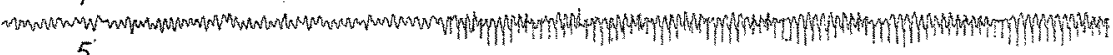

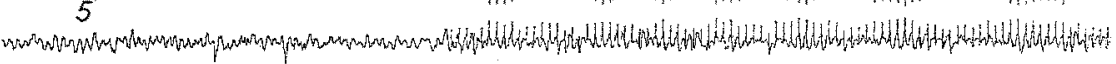
A 6

7

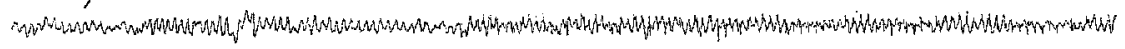

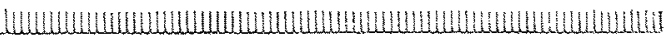

1

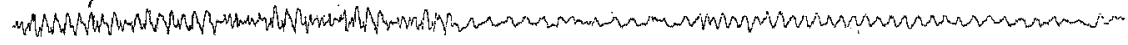
2

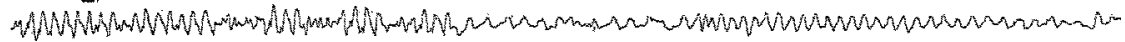

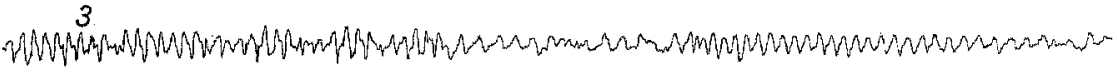
B 4

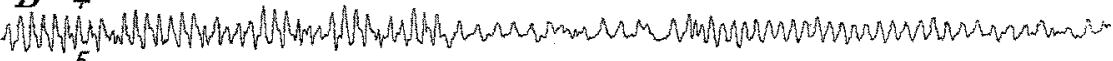

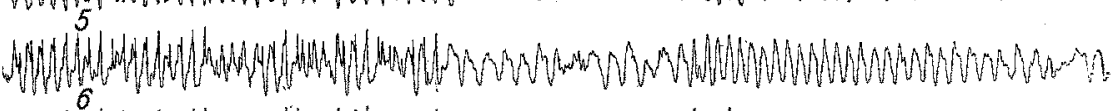

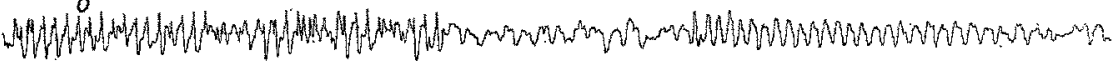

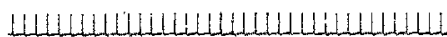

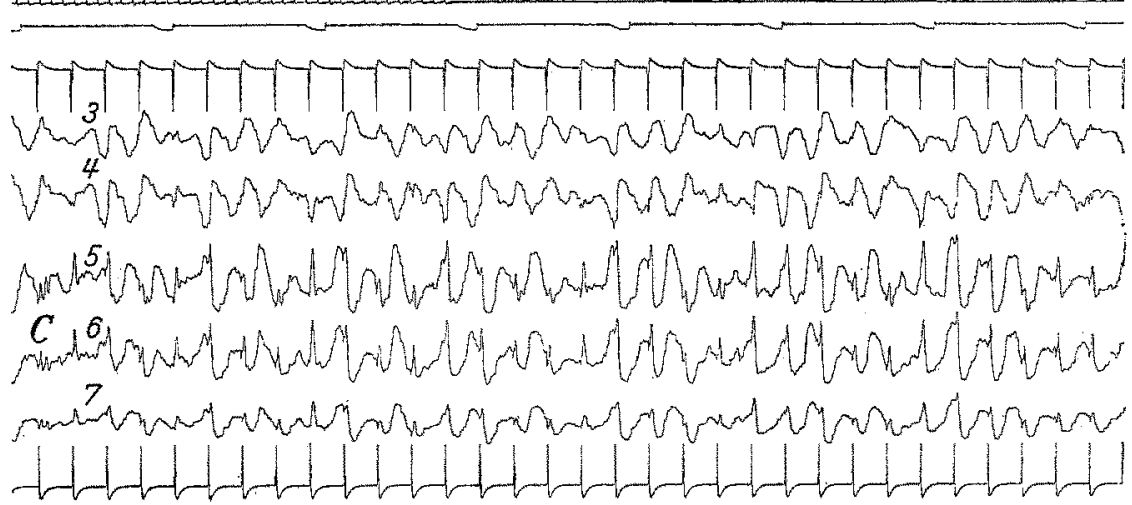

Abb.1. Schwebungen des Thetarhythmus, erzeugt durch retikuläre Reizung mit einer Frequenz, die nur wenig von der spontanen Thetafrequenz abweicht. A mit $15 \mathrm{~mm} / \mathrm{sec} ; \mathrm{B} \mathrm{mit} 30 \mathrm{~mm} / \mathrm{sec}$; $\mathrm{C} \mathrm{mit}$ 60 malsec registriert. Die arabischen Ziffern bezeichnen die Nummem dez Kontakte einer 8 fachTiefenelektrode auf gemeinsamer Trägernadel (Kontaktabstand 1,5 mm, Zähluag von oben nach unten). Unipolare Ableitungen gegen Nadelelektrode im Thalamus. Unipolare Phasenumkehr (Lage der Pyramidenzellschicht) zwischen 4 und 5 . Reizregistrierung in $A$ und $B$ im untersten Kanal, in $C$ im obersten und untersten Kanal. Eichung $200 \mu V$ 
bei einer durchschnittlichen Frequenz von 5/sec auf $2 \%$ konstant bleiben.

Es war festzustellen, ob es sich dabei um eine reine Schwebung handelt oder ob darüber hinaus evoked potentials und Thetawellen einander beeinflussen, d. $h$. ob mit den Thetawellen verbundene cyclische Schwankungen der Erregbarkeit dabei zutage treten.

Das bei retikulärer Reizung im Hippocampus auftretende evoked potential ist in seiner Form sehr inkonstant, auch wenn es in thetafreien

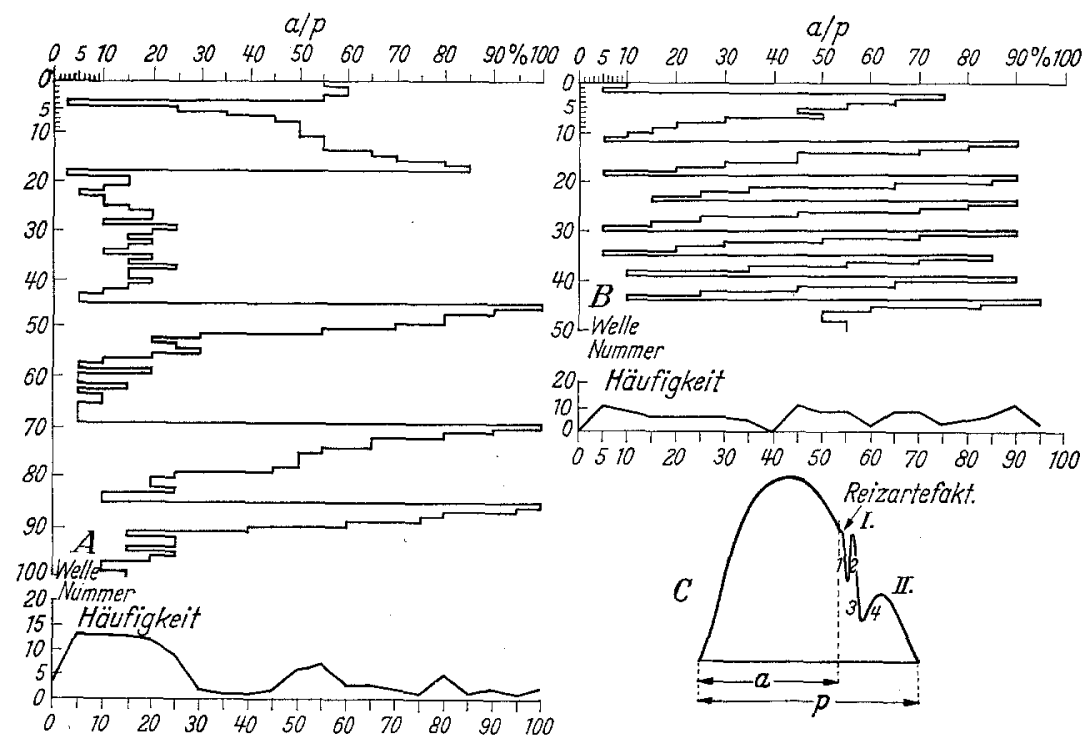

Abb.2. Sehwankungen der Thetafrequenz und deren Beeinflussung durch langsame retikuläre Reizung (näheres siehe Text). Reizintensität 13 V. $\boldsymbol{A}$ vor Eseringabe; $\boldsymbol{B}$ nach Eseringabe; $\boldsymbol{C}$ skizze der in der apikalen Dendritenschicht von $\mathrm{CA}_{\mathbf{1}}$ registrierten Thetawelie mit Überlagerung der durch die retikuläre Reizung ausgelösten Potentiale am absteigenden Schenkel. Die Abszissen der Diagramme $A$ und $B$ geben den Quotienten $a / p$ in Prozent wieder, die Ordinaten die fortlaufende Wellennummer. ohne Eserin besteht eine Tendenz der Thetawelle, mit der Reizung in Phasenkopplung aufzutreten

Intervallen gemessen wird. Es besteht vorwiegend aus zwei Komponenten, einer steileren Welle, deren Maximum in der Schicht der apikalen Dendriten elektronegativ ist und durchschnittlich eine Latenz von $22 \mathrm{msec}$ hat, und einer zweiten, langsameren, inkonstanteren Welle derselben Polarität nach 40-70 msec Latenz.

Abb. 2 zeigt die Ergebnisse eines repräsentativen Versuches, bei dem mit und ohne Eserinvorbereitung retikulär gereizt wurde. Dabei wurden je 100 aufeinanderfolgende Wellen mit $6 \mathrm{~cm} / \mathrm{sec}$ registriert und auf folgende Weise vermessen: Der Beginn des ansteigenden. Teiles der Thetawelle, in der Schicht der apikalen Dendriten, wurde für jede Welle bestimmt und die Periode der Welle $(p)$ in Millimetern gemessen. Der 
Abstand des Reizartefaktes vom Beginn der Thetawelle (a) wurde ebenfalls gemessen und in Prozent der Periode der Welle $(a / p)$ ausgedrückt. Um die Schwankungen der Thetafrequenz unter der retikulären Reizung sichtbar zu machen, wurde ein Diagramm angelegt, in dem die fortlaufende Nummer der Welle 1-100 (bzw. 1-50 unter Eserin) als Ordinate von oben nach unten aufgetragen wurde, und die dazugehörenden Phasen, in die der jeweilige Reiz fällt (also $a / p$ ), in Prozent der Thetaperiode als Abszisse. Um nicht eine höhere Meßgenauigkeit als tatsächlich erreichbar war, vorzutäuschen, wurden die Phasen, in die der Reiz fällt, in Klassen von $5 \mathrm{zu} 5 \%$ eingeteilt.

Bei dieser Darstellung wäre bei einer völlig regelmäßigen Thetafrequenz, die etwas von der Reizfrequenz abweicht, ein regelmäßiges Stufenmuster zu erwarten wie unter Eserin (rechte Hälfte der Abb.2). Ohne Eserin ist die Thetafrequenz nur zeitweise reizunabhängig, zeitweise haben jedoch die Thetawellen die Tendenz, gekoppelt mit dem Reiz aufzutreten. Diese Tendenz kommt auch in der Häufigkeitsverteilung der Phasen, in die der Reiz jeweils fällt, zum Ausdruck (Abb.2) und kann als ,resetting“ des Thetarhythmus durch retikuläre Reizung bei ungenügender eigener Frequenzkonstanz (wie es unter Eserin der Fall ist) aufgefaßt werden. Besonders, wenn der Reiz in die ersten $30 \%$ der nach negativ ansteigenden Thetawelle fällt, ist die Tendenz zur Synchronisierung von retikulär aus deutlich. Das entspricht etwa der ansteigenden Phase der Thetawelle, die kürzer ist als die absteigende.

Die einzelnen Komponenten des evoked potential in der apikalen. Dendritenregion wurden willkürlich mit Ziffern bezeichnet: 1 bildet die unmittelbar dem Reiz folgende, nach positiv gerichtete Komponente, 2 die darauffolgende nach negativ gerichtete; darauf folgt Komponente 3 , die absteigende, nach positiv gerichtete, und 4, die ansteigende, nach negativ gerichtete Komponente. $2+3$ bilden Welle I des evoked potential, 4 ist die ansteigende Flanke von Welle II, die inkonstanter und breiter ist als Welle I. Weitere Komponenten sind nicht mehr sicher zu erfassen.

Die Amplituden dieser vier Komponenten wurden in Abb. 3 graphisch als Ordinaten in Abhängigkeit von der Phase, in die der jeweilige Reiz fällt ( $a / p$ in Prozent) dargestellt. Dabei zeigte sich, daß Komponente 1, die niedrigste und nicht eindeutig vom Reizartefakt unterscheidbare, in den ersten $30-40 \%$ der Periode minimal ist. Fällt der Reiz in die absteigende Phase der Thetawelle, so ist die Amplitude dieser Komponente viel höher. Dies läßt sich dahingehend deuten, daß im ansteigenden Teil der Thetawelle die nach unten gerichtete Komponente 1 untergeht und nur im absteigenden Teil, wo sie den Abfall beschleunigt, deutlich zutage tritt. Die Amplitudenänderungen können somit als reiner Überlagerungseffekt aufgefaßt werden. 
Dasselbe trifft für Komponente $2 \mathrm{zu}$, die am deutlichsten gemessen werden konnte (siehe Skizze Abb.2). Auch die Asymmetrie des Verteilungsmusters der Komponente 2 (in Abb.3) spiegelt die Asymmetrie der Thetawelle wider, was ebenfalls als Überlagerungseffekt zu deuten ist. Welle 3 zeigt dasselbe, nur ist das Koordinatensystem, entsprechend der längeren Latenz dieser Welle, nach rechts verschoben, so daß der Gipfel der Yerteilungskurve weiter links liegt.
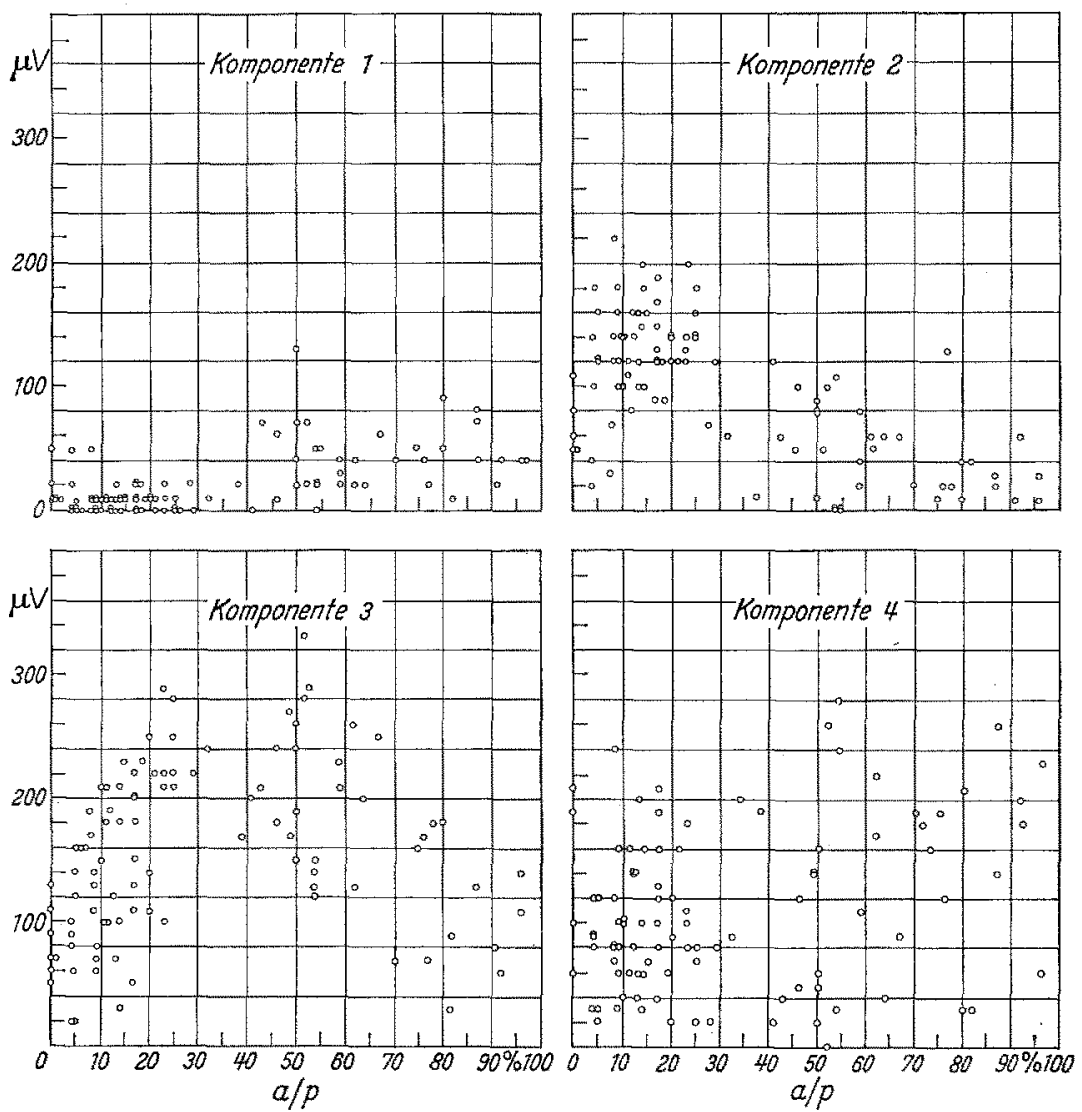

Abb.3. Amplitudendiagramm der vier enzelnen Komponenten der Reizantwort in Abhängigkeit von inrer Lage zur Thetaphase

Für Welle 4 dagegen ist keine eindeutige Verteilung mehr zu erkennen, da diese Komponente zu inkonstant in Latenz und Amplitude ist und oft kaum von der nächsten Thetawelle abgegrenzt werden kann.

Diese Messungen zeigen, daß der Thetarhythmus während retikulärer Reizung völlig unabhängig vom evoked potential seine Form bewahrt. 
H. Petsche, G. GogoláfK, Ch. Stumpe und T. Newkirk:
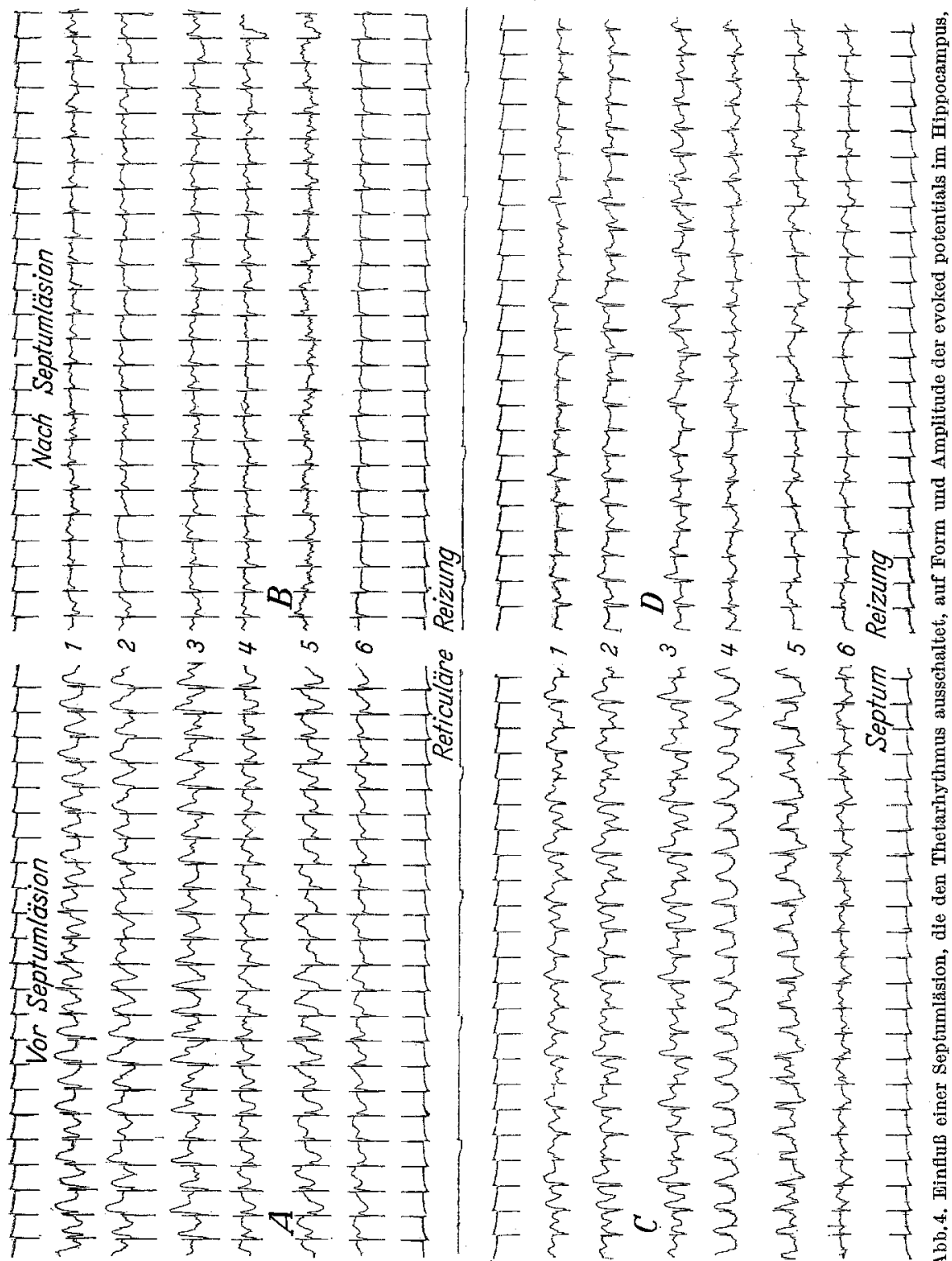

- 2 \% 3

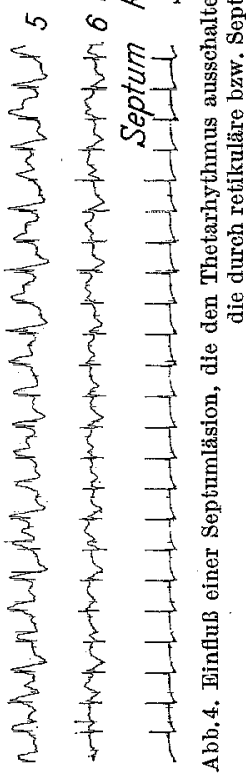

In zwei Versuchen wurde mit der achtfach-Tiefenelektrode im Hippocampus das von retikulär und das vom Septum ausgelöste evoked potential bei kontinuierlicher 5/sec Reizung verglichen und ausgemessen. Dabei läßt sich das oben als Welle I bezeichnete, in der apikalen Dendritenschicht negative Potential bei Reizung von beiden Orten aus 
identifizieren. Ihre Latenz (,,peak latency“) beträgt 22 bzw. 24,5 msec bei retikulärer, und 15 bzw. 18 msec bei Septumreizung (GLOor u. Mitarb. 1963, beschreiben dasselbe Phänomen als Komponente I des evoked potential im Katzenhippocampus und finden bei Stimulation des N. amygdalae Latenzen zwischen 20 und $43 \mathrm{msec}$, bei entorhinaler Reizung 20-30 msec). Für die Distanz zwischen retikulärem Reizort und Septum ist somit eine Reizleitungszeit von 7 bzw. 6,5 msec anzunehmen. Die zweite Komponente konnte, da inkonstant, nicht verwertet werden.

Abb.4 zeigt das Verhalten des evoked potential, von retikulär und vom Septum ausgelöst, vor und nach Septumkoagulation, die zu einer völligen Unterdrückung des Thetarhythmus geführt hat. Die linke Hälfte der Abbildung zeigt den Unterschied im Verhalten des Thetarhythmus, der bei retikulärer Reizung in seiner Frequenz unbeeinflußt bleibt, wodurch das evoked potential (dessen Frequenz etwas von der Thetafrequenz abweicht) über ihn wandert. Bei Septumreizung dagegen (untere Hälfte der linken Seite der Abb.4) wird der Thetarhythmus synchronisiert, besonders deutlich in Kanal 5. Nach Koagulation ist der Theta praktisch aufgehoben und die evoked potentials, sowohl nach retikulärer als auch nach Septumreizung, erscheinen überdies stark abgeschwächt. Dies ist ein weiterer Hinweis dafür, daß die Bahnen, die die zum evoked potential führenden Erregungen leiten, zum größten Teil durch die mediane Septumregion ziehen.

\section{Diskussion}

Es wird allgemein angenommen, daß jene Bahnen, die für das Auftreten des Thetarhythmus verantwortlich sind, vom Mittelhirn über das Septum in den Hippocampus verlaufen (STUMPF u. Mitarb. 1962). Nach den anatomischen Gegebenheiten ist zu erwarten, daß eine elektrische Reizung der Formatio reticularis des Mittelhirns und das Septums mit Einzelreizen, bzw. niederfrequenten Reizserien im Hippocampus ähnliche Reizantworten auslöst.

Wie die in dieser Arbeit mitgeteilten Ergebnisse zeigen, trifft dies auch zu. Erwartungsgemäß haben die retikulär ausgelösten Hippocampusreizantworten auch längere Latenzen, als wenn sie vom Septum ausgelöst werden. Trotz der weitgehend ähnlichen Konfiguration beider Reizantworten bestehen folgende deutliche Unterschiede:

a) Bei niederfrequenter Septumreizung ,folgen“ die evoked potentials im Hippocampus innerhalb eines relativ weiten Frequenzbandes, etwa zwischen 4 und 30/sec, wobei die obere Grenze stark schwankt. Im Gegensatz dazu läßt sich die spontane Thetafrequenz durch langsame Mittelhirnreizung nur in engen Grenzen beeinflussen. Reizungen mit höheren 
Frequenzen führen rasch zum unspezifischen Effekt einer arousal reaction, mit Aktivierung einer raschen, frequenzstabilen Thetaaktivität.

b) Während niederfrequenter Septumreizung ist die spontane Thetatätigkeit praktisch völlig unterdrückt; die über diesem flachen Hintergrund auftretenden rhythmischen evoked potentials täuschen nur die Aktivierung eines Thetarhythmus vor. Im Gegensatz dazu verschwindet der spontane Theta bei langsamer Vittelhirnreizung nicht, sondern die dabei entstehenden evoked potentials überlagern sich ihm zu den oben beschriebenen Schwebungen.

c) Die durch niederfrequente Septumreizung im Hippocampus ausgelösten Reizantworten sind pharmakologisch nur äußerst schwer zu beeinflussen. Von zahlreichen, seinerzeit auf diesen Effekt bin untersuchten Substanzen (BRËCKE, Gogoxák u. STUMPF 1961) hatte lediglich LSD eine deutliche Wirkung. Im Gegensatz dazu ist der Effekt einer Mittelhirnreizung einer pharmakologischen Beeinflussung leicht zugänglich (BRt̛cke, SATLER u. STuMPF 1957). Nach Verabreichung von Substanzen, die die Frequenz des Thetarhythmus herabsetzen, z. B. nach Hexobarbital, sinkt auch die optimale Frequenz für Schwebungen in entsprechender Weise.

Diese Unterschiede lassen den Schluß zu, daß eine niederfrequente Septumreizung den Hippocampus viel intensiver beeinflubt als eine niederfrequente Mittelhirnreizung. Trotzdem scheinen diese Unterschiede nicht prinzipieller Natur zu sein. Sie können auf folgende Weise gedeutet werden: Innerhalb der Septumregion ist nur ein kleiner Bereich für die Auslösung des typischen Reizeffektes im Hippocampus verantwortlich. Bei Messungen der Schwelle für die Thetaausschaltung steigen die Schwellenspannungen rasch an, wenn die Reizelektrode rom optimalen Bereich entfernt wird. Vermutlich ist dieser Bereich mit jenem Kerngebiet identisch, in dem die Zellen gefunden wurden, die dem Thetarhythmus synchron gruppiert entladen (PETscHe u. Mitarb. 1962). Es ist demnach wahrscheinlich, daß bei elektrischer Reizung an dieser Stelle das gesamte verantwortliche Zellareal gereizt wird. Das Gebiet des Mittelhirnes dagegen, bei dessen Reizung Antworten im Hippocampus auftreten, ist unverhältnismäBig groß und offenbar mit jenem Gebiet identisch, das als ascendierendes retikuläres System bezeichnet wird. Elektrische Reizung in diesem Gebiet kann daher nur einen kleinen Anteil jener Neuronen, die mit dem Septum in Verbindung stehen, erregen. Der Strom von Impulsen aus den durch die Reizung nicht beeinflußten Neuronen jener Region genügt aber, die Thetatätigkeit im Hippocampus aufrechtzuerhalten. Somit ist der beschriebene Überlagerungseffekt zu erwarten.

Dieselben Ubberlegungen lassen auch das Phänomen des, ,resetting" des Thetarhythmus erklären. Darunter ist folgendes zu verstehen: Es 
gelingt unter bestimmten Umständen, durch einen Reiz die rhythmische Periodik des Thetarhythmus zu unterbrechen, worauf der Rhythmus neu geordnet wird, d. h. die Zeitdifferenz zwischen zwei Wellenkuppen, eine vor und eine nach dem Reiz, entspricht keinem ganzzahligen Vielfachen der Thetaperiode. Vom Septum aus ist dieses Phänomen nicht zu erzielen: Das evoked potential eines einzelnen Reizes überlagert sich der Thetatätigkeit, ohne ihre Periodik zu stören, was auch verständlich ist, wenn man bedenkt, daß für die Periodik in erster Linie der Strom von Impulsen aus der Formatio reticularis verantwortlich ist. Werden dagegen Einzelimpulse retikulär gegeben, so kann es bei schwach ausgeprägtem Thetarhythmus zu einer vorübergehenden Phasenkopplung von Reiz- und Thetafrequenz kommen (wie in Abb.2 gezeigt wurde), als Zeichen dafür, daß sich in diesem Fall der Thetarhythmus vorübergehend von retikulär aus steuern läßt. Bei besser ausgeprägtem Thetarhythmus, etwa unter Eserin, versehwindet dieses Phänomen und die Thetafrequenz läßt sich durch Einzelreize oder langsame rhythmische Reizung nicht mehr beeinflussen. Vermutlich läßt sich diese Erscheinung so deuten, daß bei schwachem, inkonstanten Thetarhythmus nur einige Neuronen der Formatio reticularis zum Zustandekommen des Thetarhythmus beitragen, und ihre Tätigkeit somit leichter durch Reizung beeinflußt werden kann, als wenn die Formatio reticularis durch Eserin in einen Zustand erhöhter Erregung gesetzt wird.

Nicht völlig geklärt werden konnte die Frage, ob für alle Effekte einer Mittelhirnreizung auf den Hippocampus die Integrität des Septums erforderlich ist. Bekanntlich ist nach einer medianen Septumläsion ein Thetarhythmus nicht mehr auslösbar, obwohl an solchen Tieren eine hochfrequente Mittelhirnreizung das Hippocampus-EEG immer noch, wenn auch nur mehr im Sinne einer Frequenzzu- und Amplitudenabnahme, verändert. Es besteht somit noch die Möglichkeit, daß die Abflachung des arhythmischen Hippocampus-EEGs beim septumlädierten Kaninchen nach hochfrequenter retikulärer Reizung über andere Hippocampusafferenzen zustande kommt. Daß für diese beiden Phänomene, die Thetaaktivierung einerseits und die Hippocampus-EEG-Abflachung andrerseits, die beim erwachsenen Tier als Ausdruck einer arousal reaction gleichzeitig vorkommen und sich nicht trennen lassen, verschiedene Systeme verantwortlich sein dürften, die auch zu verschiedenen Zeiten ausreifen, zeigten unsere Untersuchungen über die Entwicklung des Thetarhythmus (GoGolák u. Mitarb. 1963): Die Abflachung als Ausdruck einer arousal reaction kann schon bald nach der Geburt beobachtet werden, wogegen die Thetaaktivierung erst um den 12. Lebenstag auftritt.

Septumläsionen, die zur Thetaunterdrückung führen, bewirken auch eine erhebliche Reduzierung der durch niederfrequente Mittelhirnreizung. 
im Hippocampus ausgelösten Reizantworten. Trotzdem kann nicht gesagt werden, daß dieser Reizeffekt durch eine Septumläsion völlig aufgehoben würde, und es besteht durchaus die Möglichkeit, daß ein Teil der zum Zustandekommen des evoked potentials erforderlichen Impulse über andere Afferenzen in den Hippocampus gelangt.

\section{Zusammenfassung}

Langsame rhythmische elektrische Reizungen der medianen Septumregion und der Formatio reticularis des Mittelhirns lösen im Hippocampus des Kaninchens evoked potentials aus, die in ihrer Konfiguration ähnlich sind und sich vornehmlich durch ihre Latenz unterscheiden. Die vergleichbare Form, die Latenzunterschiede und der Umstand, daß sie durch mediane Septumläsion in ihrer Amplitude weitgehend vermindert werden, lassen den Schluß zu, daß auch die von retikulär ausgelösten, im Hippocampus registrierten evoked potentials über das Septum geleitet werden.

Der Effekt langsamer rhythmischer Reizung von beiden Reizorten auf den Thetarhythmus ist allerdings verschieden. Bei Septumreizung kommt es zu einer rhythmischen Folge von evoked potentials bei unterdrücktem Thetarhythmus (früher als ,Theta driving“ bezeichnet); bei retikulärer Reizung dagegen treten rhythmische Schwebungen der Thetaamplitude auf, deren Frequenz der Differenz zwischen Thetafrequenz und Reizfrequenz entspricht. Durch Messung der Abhängigkeit der Amplitude der einzelnen Komponenten des evoked potential von der Thetaphase konnte gezeigt werden, daß es sich dabei um einen rein physikalischen Überlagerungseffekt handelt und keine Beeinflussung durch einen Frregbarkeitscyclus anzunehmen ist.

Der verschiedenartige Effekt von Septum- und retikulärer Reizung auf den Hippocampus-Thetarhythmus wird darauf zurückgeführt, daß bei der ersteren in einem örtlich streng umschriebenen pacemaker für den Thetarhythmus gereizt wird, bei der letzteren dagegen innerhalb einer weiten, nicht exakt abgrenzbaren Region, der Formatio reticularis, die mit dem Hippocampus in Verbindung steht und von der immer nur ein relativ umschriebener Teil durch die Reizung erregt werden kann.

Ferner wurde nachgewiesen, daß ein ,,resetting" des Thetarhythmus nur bei relativ instabilem Theta und nur von retikulär aus zu erzielen ist.

\section{Literatur}

Brücke, F., G. Goqolák u. Ch. StumpF: Die Wirkung von LSD auf die Makround Mikrotätigkeit des Hippocampus. Naunyn-Schmiedeberg's Arch. exp. Path. Pharmak. 240, 461-468 (1961).

- H. Petsche, B. Plllat u. E. Deisenhaminer: Über Veränderungen des Hippocampus-Elektroencephalogrammes beim Kaninchen nach Novocaininjektion in die Septumregion. Naunyn-Schmiedeberg's Arch. exp. Path. Pharmak. 237, 276-284 (1959a). 
Brücke, F., H. Petsche, B. Pillat u. E. Detsenhammer: Die Beeinflussung der „Hippocampus-arousal-reaction“ beim Kaninchen durch elektrische Reizung im Septum. Pflügers Arch. ges. Physiol. 269, 319-338 (1959b).

- S. SATLER u. CH. STUMPF: Pharmakologische Beeinflussung der Frequenz der Hippocampustätigkeit während retikulärer Reizung. Naunyn-Schmiedeberg's Arch. exp. Path. Pharmak. 281, 267-278 (1957).

Chang, H. T.: Changes in excitability of cerebral cortex following a single electric shock applied to the cortical surface. J. Neurophysiol. 14, 95-111 (1951).

GLOOR, P., C. L. VERA and L. SPERTI: Electrophysiological studies of hippocampal neurons. I-II. Electroenceph clin. Neurophysiol. 15, 353-402 (1963).

Gogolák, G., H. Petsche u. Сh. StumpF: Die Entwicklung des HippocampusThetarhythmus beim Kaninchen. Pflügers Arch. ges. Physiol. 277, 166-177 (1963).

GREEN, J.D., and A. ARDUINI: Hippocampal electrical activity in arousal. J. Neurophysiol. 17, 533-557 (1954).

- D.S. MAXWELL, W. J. SchindeER and CH. StumpF: Rabbit's EEG "theta" rhythm: its anatomical source and relation to activity in single neurons. J. Neurophysiol. 28, 403-420 (1960).

Petsche, H., G. Gogolák and CH. Stumpt : The significance of the rabbit's septum as a relay station between the midbrain and the hippocampus. I. Electroenceph. clin. Neurophysiol. 14, 202-211 (1962).

SAILER, S., u. Ch. StunPF: Beeinflußbarkeit der rhinencephalen Tätigkeit des Kaninchens. Naunyn-Schmiedeberg's Arch. exp. Path. Pharmak. 231, 63-77 (1957).

Stumpr, Ch., H. Petsche and G. Gogorák: The significance of the rabbit's septum as a relay station between the midbrain and the hippocampus II. Electroenceph. clin. Neurophysiol. 14, 212-219 (1962).

Dozent Dr. H. Petsche,

Neurologisches Institut der Universität Wien, Wien IX, Österreich, Schwarzspanierstraße 17 